\title{
VEGETATION DYNAMICS ALONG AN ELEVATIONAL GRADIENT IN TERICH VALLEY, CHITRAL HINDUKUSH RANGE, NORTHERN PAKISTAN
}

\author{
ZAMAN, A.* - BADSHAH, L. \\ Phytoecology Lab, Department of Botany, University of Peshawar, Peshawar, Pakistan \\ (phone: +92-345-319-7085 - Zaman, A.; +92-333-894-4128 - Badshah, L.) \\ *Corresponding author \\ e-mail: akhtarzaman@uop.edu.pk \\ (Received 25 $5^{\text {th }}$ Mar 2020; accepted $9^{\text {th }}$ Jul 2020)
}

\begin{abstract}
This phytosociological study was carried out during 2016-2019 in Terich valley, Hindukush range, Chitral Pakistan. The objective of this study was to determine the vegetation dynamics along various ecological parameters. The quantitative data of vegetation were collected from five monitoring sites using the systematic-random sampling quadrate method. The size of quadrates was $10 \mathrm{~m}^{2}$ for the trees, $6 \mathrm{~m}^{2}$ for shrubs and $1.5 \mathrm{~m}^{2}$ for herbaceous species. Three communities viz. Elaeagnus-PrunusAdiantum, Fraxinus-Rosa-Acanthophylum and Hippophae-Sophora-Poa community structures were established at foot-hills. Mid-hills comprised Ferula-Artemisia-Capparis, Prangos-Ribes-Berberis and Astragalus*-Astragalus-Eremurus communities. Community structure was quite different at sub-alpine (Artemisia-Rhodiola-Rosularia, Betula-Scutellaria-Taraxacum) and alpine zones (AnaphalisCousinia-Kobresia, Alajja-Oxyria-Oxytropis). Soil samples were collected and analyzed to study soil properties. Soils were slightly acidic and had low content of organic matter. Vegetation was found to be under extreme biotic stress. Results also showed that vegetation at higher elevation showed lower species richness.
\end{abstract}

Keywords: phytosociology, edaphic parameters, similarity index, species diversity, species richness, maturity index, dry temperate vegetation

\section{Introduction}

Vegetation is an ecological expression of plants growing together in a specific area (Wahab et al., 2010). Vegetation structure depends upon the prevailing environmental conditions (Khan et al., 2011). Plant community is the collection of functional units of species both spatially and temporally. The changes in the community structure are due to seasonal variations and the sampling time (Forzieri et al., 2011; Ali et al., 2018; Hussain et al., 2019). Therefore, the community structure reflects seasonal aspects. The key environmental factors influencing the makeup of the community include over-grazing, deforestation, trampling, construction of buildings, soil erosion, chopping and other natural factors (Ali and Malik, 2010). Vegetation is also influenced by atmospheric humidity, soil moisture, aspect and grazing intensity (Ahmad, 2009; Shaheen and Qureshi, 2011). Vegetation plays a significant role in ecosystem composition and worldwide water balance (Kucharik et al., 2000). Soil is the main factor that determines the different features of vegetation species in particular area (Khan et al., 2010a). Physio-chemical properties of the soil also influence the plants distribution pattern on small scale (Bakkenes et al., 2002). Topography is also an important determinant of vegetation structuring as it alters other environmental factors like temperature, humidity etc. Difference in altitudes, slope and aspect also support different communities (Arora, 2002). The present study was conducted in Terich Valley Chitral of Hindukush range. Five monitoring sites were established viz. Shagrom, Warimon, Zondrangam, Rosh Gol 
and Ghari. On the basis of importance values (IV) 10 different plant communities were established at monitoring site. Different environmental factors such as temperature, soil type, topography and biotic factors influence the composition and the distribution of plant communities in the investigated area. A lot of quantitative phytosociological work has been published from various parts of Pakistan. Ahmed et al. (2006) surveyed different climatic regions of Himalaya and recorded 4 mono-specific forests for analyses of vegetation. Ali et al. (2007) described plants associations and other ecological characteristics of rangelands of Dir. Khan et al. (2010b) observed the physiognomy of Monotheca buxifolia forest in Lower Dir with altered elevations and established 6 species associations with Monotheca buxifolia. Rashid et al. (2011) recorded the communities structure of Malam Jabba, swat. Ahmed et al. (2011) investigated forests of Cedrus deodara in Himalayan and Hindukush mountainous range. Khan et al. (2012) documented the vegetation pattern of forested regions of Chitral. Khan (2012) explored the community structure of Quercus species in district Dir. Data were analyzed by Point Centered Quarter method, PC-Ord for cluster classification and Dentrended correspondence analysis for ordination of edaphic and environmental factors. While the major types of Quercus sp. vegetation associations were also assessed. Khan et al. (2010c) explored the vegetation-environment relationship of Chitral applying Point Centered Quadrate technique. Mohib Shah (2013) surveyed the phytodiversity and vegetation-environment relationship of Peer Taab graveyard (Swabi). Muhammad et al. (2015) worked on vegetation structure of Acacia modesta at various elevational values in Malakand division. Rahman et al. (2016) recorded vegetation composition of Isodon rugosus communitie of Khwaza khela, Swat. Khan et al. (2016) worked on pines' forests and their phytosociology in Indus-Kohistan. A total of six groups and four specific plots of Cedrus deodara were enlisted. Irshad et al. (2016) recorded the stress on environment about scattering pattern of different species of Punica granatum community in district Lower Dir. Ali et al. (2018) documented 15 plant communities, 5 for shrubs, 5 for herbs and 5 for trees of Hindukush Range Swat Pakistan. Ilyas et al. (2018) worked on Kabal Valley, Swat Pakistan to describe the vegetation structures of the sub- tropical range. Hussain et al. (2019) quantified different aspects of vegetation in Koh-e-Safaid Range, Upper Kurram, Pakistan, and summed up seven plant communities of trees, shrubs and herbs, and no studies have addressed the vegetation structure of Terich valley, Chitral a series in the famous Hindukush Mountain range in Pakistan. Being situated on "ecotone zone" this region exhibits special geological features and hence special sort of vegetation distribution. The present study in this region was therefore, aimed to document vegetation structure and characteristics species to inform future conservation strategies.

\section{Materials and methods}

\section{Study area}

Terich valley is a charming valley of Chitral, Pakistan which provides a wide range of habitat to wild flora. It is located $72^{\circ} 07^{\prime}$ to $73^{\circ} 97^{\prime}$ E longitude and $35^{\circ} 20^{\prime}$ to $36^{\circ}$ $55^{\prime} \mathrm{N}$ latitude in Chitral. It is bounded on the North by Tajikistan, on the West by Badakhshan, on the South by Nuristan while on the East by District Ghizer of GilgitBaltistan (Fig. 1). Rugged and uneven terrain characterizes the valley. The climate is cold temperate type. Temperature ranges from $-12{ }^{\circ} \mathrm{C}$ in winter to $30{ }^{\circ} \mathrm{C}$ in summer. Phytogeographically, Terich valley lies in the Irano-Turranian floristic region. 
Floristically, the Irano-Turranian region is luxuriant occupied $45.6 \%$ of the flora of Pakistan. In Terich valley, Terich Mir $(7685 \mathrm{~m})$ the highest peak of the Hindukush range is followed by Saraghrar (7349 m), Shakawar (7116 m), Langar Peak (7100 m) and many other peaks with low altitudes.
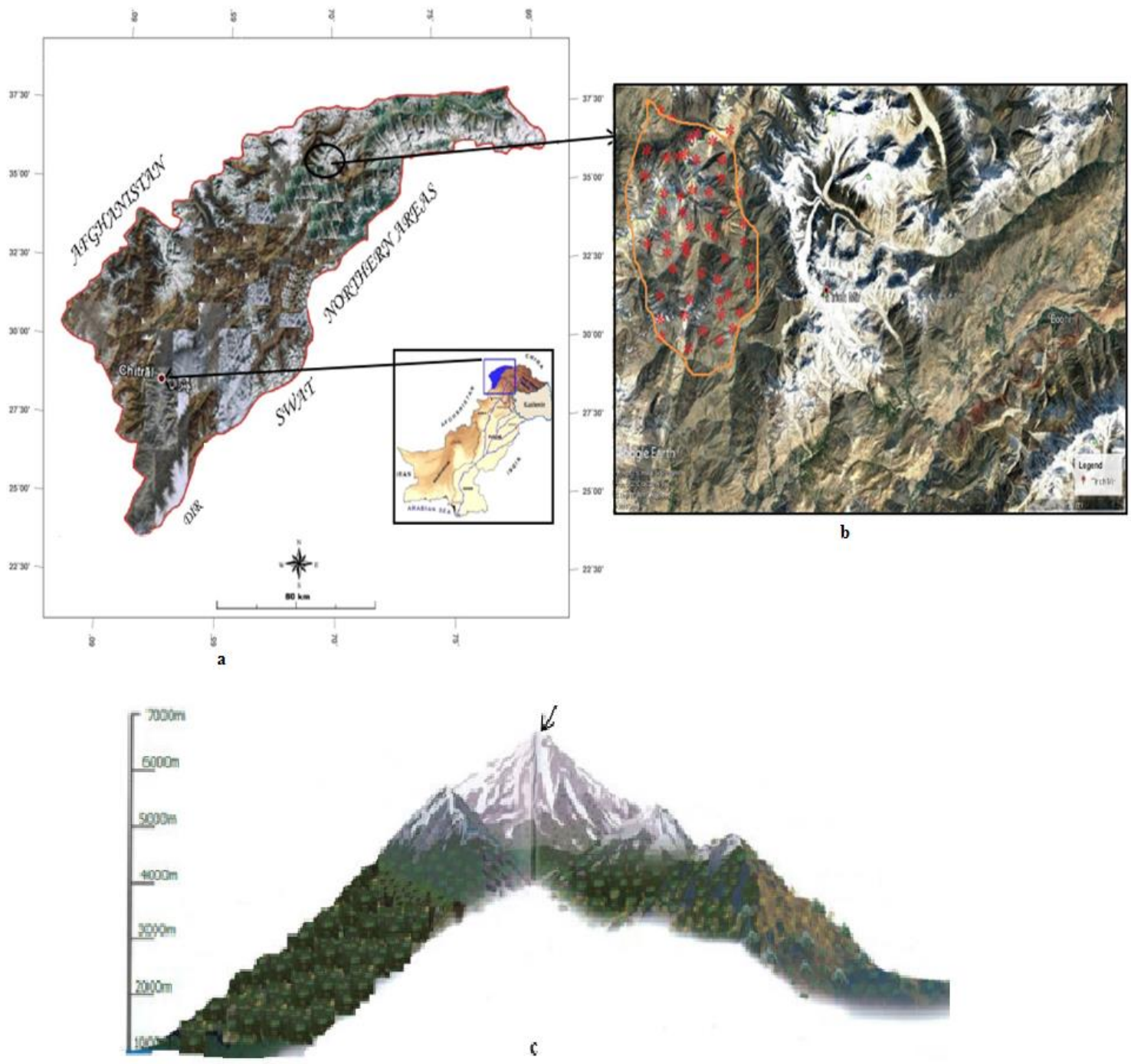

Figure 1. a) Geo-reference Map of Chitral, b) The study area showing the sampling sites, $c$ ) View of Research area along Terich Mir

\section{Phytosociology}

Phytosociological studies were conducted in 5 monitoring sites (Table 1). These sites were selected based on altitude, topography, species composition and physiognomic features. Vegetation was evaluated by using $15\left(1.5 \mathrm{~m}^{2}\right)$ quadrats for herbs, $10\left(5 \mathrm{~m}^{2}\right)$ quadrats for shrubs, $5\left(10 \mathrm{~m}^{2}\right)$ quadrats for trees at each monitoring site. Phytosociological attributes viz. Density, cover and frequency of each species was measured and values were changed to relative values to calculate IV (Importance value). The plant communities were established based on highest importance values following (Hussain, 1989; Ahmad and Shaukat, 2012). 
Table 1. Locations, geographical and ecological characteristics of the sampling sites

\begin{tabular}{|c|c|c|c|c|c|c|}
\hline Main Location & Altitude (m) & Slope $\left(^{\circ}\right)$ & Aspect & Soil type & GPS Coordinates & Vegetation \\
\hline Shagrom & $1000-2500$ & $\begin{array}{l}12 \\
17\end{array}$ & $\begin{array}{c}\text { Foot \& } \\
\text { Mid-hills }\end{array}$ & \multirow{5}{*}{$\begin{array}{l}\text { Sandy } \\
\text { loam }\end{array}$} & $\begin{array}{l}71.41 \\
35.54\end{array}$ & Herbaceous \\
\hline Warimon & $1600-2420$ & $\begin{array}{l}25 \\
29 \\
\end{array}$ & $\begin{array}{c}\text { Foot \& } \\
\text { Mid-hills }\end{array}$ & & $\begin{array}{l}71.46 \\
35.53 \\
\end{array}$ & Scrubby \\
\hline Zondrangam & $1760-2350$ & $\begin{array}{l}15 \\
32 \\
\end{array}$ & $\begin{array}{c}\text { Foot \& } \\
\text { Mid-hills }\end{array}$ & & $\begin{array}{l}71.05 \\
35.45 \\
\end{array}$ & Scrubby \\
\hline Rosh Gol & $3440-3650$ & $\begin{array}{l}16 \\
33\end{array}$ & $\begin{array}{c}\text { North } \\
\text { facing \& } \\
\text { South } \\
\text { facing }\end{array}$ & & $\begin{array}{l}71.41 \\
35.53\end{array}$ & Sub-alpine \\
\hline Ghari & $2950-4200$ & $\begin{array}{l}27 \\
22\end{array}$ & $\begin{array}{c}\text { North } \\
\text { facing \& } \\
\text { South } \\
\text { facing }\end{array}$ & & $\begin{array}{l}71.41 \\
35.41\end{array}$ & Alpine \\
\hline
\end{tabular}

\section{Species diversity}

Species diversity was obtained by Hussain (1989) method.

$$
\text { Diversity Index (D) }=\frac{\sum \mathrm{n}(\mathrm{n}-1)}{\mathrm{N}(\mathrm{N}-1)}
$$

where,

$\mathrm{D}=$ Simpson index,

$\mathrm{N}=$ Number of individuals of all species,

$n=$ Number of individuals of a species.

The obtained value was subtracted from 1 to obtained Diversity Index.

\section{Species richness}

Species richness was calculated after Menhinick (1964) using the following formula.

$$
d=\frac{S}{\sqrt{N}}
$$

where,

$\mathrm{d}=$ Species richness,

$\mathrm{S}=$ Total number of species in a stand,

$\mathrm{N}=$ Total number of individuals in a stands.

\section{Similarity index}

Similarity index was calculated by using Sorensen's index (Sorensen, 1948) as modified by Motyka et al. (1950).

$$
I s_{m o}=\frac{2 W}{A+B} \times 100
$$


where,

Is = index of similarity of Motyka,

$\mathrm{W}=$ Sum of the lowest quantitative value of species common to both stands A and B,

$A=$ Sum of the quantitative value of all species in stands/community $A$,

$\mathrm{B}=$ Sum of the quantitative value of all species in stand/community $\mathrm{B}$.

\section{Maturity index}

Community maturity index was obtained by Pichi-Sermolli (1948) method.

$$
\text { Degree of maturity index }=\frac{F t}{N}
$$

where,

$\mathrm{Ft}=$ Frequency values of all species in a stand,

$\mathrm{N}=$ Total number of species in a stand.

Importance value index (IVI)

The ecological importance of a species in relation to the community structure was obtained by adding the relative values of density, frequency and cover.

The equation used for the calculation of IVI is given as under:

$$
\text { Importance value index }(\mathrm{IVI})=\frac{\mathrm{D} 3+\mathrm{C} 3+\mathrm{F} 3}{3}
$$

\section{Soil analysis}

About $1 \mathrm{~kg}$ soil sample (upto a depth of $15 \mathrm{~cm}$ ) was taken from each five localities of Terich valley. The collected samples were then mixed to get a composite sample, kept in polythene bags and taged appropriately for analyses in the research laboratory. The soil samples analyses were carried out at Pakistan Tobacco Board, Mardan for determination of physio-chemical properties. Soil Texture triangle was used for the identification of texture classes (Bouyoucos, 1936; Brady, 1990). Soil pH was noted by using 1:5 soil water suspensions (Black, 1965). For quantification of organic matter in soil followed Walkley (1947). Lime \% (CaCO3) was determined by acid base nutrilization following Rayan et al. (1997). Nitrogen concentration was estimated with Kjeldahl methodology (1983) and $\mathrm{Na}$ Concentration was examined by using flame photometery. Potassium and Phosphorus contents were quantified following Olsen and Sommers (1982).

\section{Results}

Present study was conducted in Terich valley, Chitral Hindukush range Northern Pakistan during 2016-19. Five selected sites viz. Shagrom, Warimon, Zondrangam, Rosh Gol and Ghari were established. The study area shows a distinctive floristic composition and vegetation pattern. A total of 195 species were documented in five sampling sites. Based on importance value (IV) at Shagrom, Warimon, Zondrangam, Rosh Gol and Ghari from each monitoring sites two communities each were recorded (Table 2). The vegetation of the area was mostly scrubby, characterized by herbaceous, shrubby plants and rarely trees. 
Table 2. Importance value data for plant species

\begin{tabular}{|c|c|c|c|c|c|c|}
\hline S.No & Plant Species & Site I & Site II & Site III & Site IV & Site V \\
\hline A. & \multicolumn{6}{|c|}{ Trees } \\
\hline 1. & Betula chitralica Browicz & 0 & 0 & 0 & 27.21 & 0 \\
\hline 2. & Betula utilis D.Don & 0 & 0 & 0 & 0 & 17.75 \\
\hline 3. & $\begin{array}{c}\text { Cotoneaster affinis var. bacillaris (Lindl.) } \\
\text { Schneider }\end{array}$ & 0 & 12.06 & 8.19 & 0 & 0 \\
\hline 4. & Crataegus songarica $\mathrm{C}$. Koch. & 0 & 14.42 & 7.56 & 0 & 0 \\
\hline 5. & Crataegus wattiana Hemsl. \& Lace, J .L. & 0 & 0 & 0 & 0 & 11.07 \\
\hline 6. & Elaeagnus angustifolia var. angustifolia & 24.31 & 7.57 & 10.93 & 0 & 0 \\
\hline 7. & Fraxinus xanthoxyloides (G. Don) DC. & 11.66 & 19.63 & 0 & 0 & 0 \\
\hline 8. & Linaria odora (M.B.) Fisch. & 0 & 0 & 8.65 & 0 & 0 \\
\hline 9. & Pistacia atlantica subsp. cabulica & 0 & 0 & 0 & 15.83 & 0 \\
\hline 10. & Prunus griffithii (Boiss.) C.K.Schneid & 10.16 & 9.53 & 0 & 0 & 0 \\
\hline 11. & Prunus jacquemontii Hook.f. & 19.99 & 0 & 0 & 0 & 0 \\
\hline 12. & Prunus prostrata Labill. & 7.81 & 0 & 0 & 0 & 0 \\
\hline 13. & Salix pycnostachya Andersson. & 8.98 & 0 & 0 & 0 & 0 \\
\hline B. & \multicolumn{6}{|c|}{ Shrubs } \\
\hline 14. & Berberis calliobotrys Aitch.ex Koehne. & 0 & 20.74 & 0 & 0 & 0 \\
\hline 15. & Berberis lyceum Royle. & 18.58 & 0 & 0 & 0 & 0 \\
\hline 16. & Berberis parkeriana Schneid. & 0 & 11.91 & 0 & 0 & 0 \\
\hline 17. & Buxus wallichiana Baill, Monogr. & 13.29 & 0 & 0 & 0 & 0 \\
\hline 18. & Clematis orientalis $\mathrm{L}$. & 8.66 & 0 & 0 & 0 & 0 \\
\hline 19. & $\begin{array}{l}\text { Cotoneaster racemiflorus (Desf.) Booth ex } \\
\text { Bosse }\end{array}$ & 15.3 & 0 & 0 & 0 & 0 \\
\hline 20. & Cousinia pycnoloba Boiss. & 0 & 0 & 0 & 0 & 25.74 \\
\hline 21. & Hippophae rhamnoides Rousi. & 0 & 0 & 17.36 & 0 & 0 \\
\hline 22. & Juniperus communis L. & 1.71 & 0 & 0 & 0 & 0 \\
\hline 23. & Lonicera myrtillus Hook. f. \& Thoms. & 0 & 0 & 7.44 & 0 & 0 \\
\hline 24. & Rhamnus prostrata Jacq.ex Parker & 0 & 11.03 & 9.74 & 0 & 0 \\
\hline 25. & Rosa beggeriana Schrenk. & 0 & 11.81 & 11.81 & 0 & 0 \\
\hline 26. & Rosa ecae Aitch. & 0 & 0 & 0 & 16.10 & 0 \\
\hline 27. & Rosa webbiana Wall.ex. Royle. & 0 & 17.93 & 0 & 0 & 0 \\
\hline 28. & Sophora mollis subsp. duthiei (Prain) Ali & 0 & 0 & 13.86 & 0 & 0 \\
\hline 29. & Tamaricaria elegans (Royle) Qaiser \& Ali & 0 & 13.32 & 10.77 & 0 & 0 \\
\hline C. & \multicolumn{6}{|l|}{ 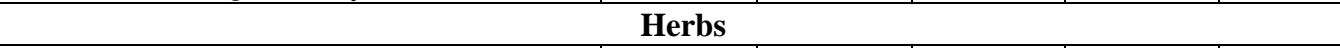 } \\
\hline 30. & Acantholimon leptostahyum Aitch. & 0 & 0 & 0 & 14.45 & 0 \\
\hline 31. & Acantholimon longiflorum Boiss. & 0 & 0 & 0 & 14.73 & 0 \\
\hline 32. & Acanthophylum laxiflorum Boiss. & 0 & 17.91 & 0 & 0 & 0 \\
\hline 33. & Adiantum venustum D. Don & 19.66 & 0 & 0 & 0 & 0 \\
\hline 34. & Alajja rhomboidea (Benth.) Ikonn. & 0 & 0 & 0 & 0 & 24.63 \\
\hline 35. & Allium caroliniannum DC. & 0 & 0 & 0 & 10.66 & 0 \\
\hline 36. & Allium chitralicum Wang \& Tang & 12.88 & 0 & 0 & 0 & 0 \\
\hline 37. & Aloitis smithii Omer. & 0 & 0 & 0 & 0 & 18.95 \\
\hline 38. & Amaranthus viridis $\mathrm{L}$. & 0 & 12.45 & 0 & 0 & 0 \\
\hline 39. & Anethum gravelons L. & 15.61 & 0 & 0 & 0 & 0 \\
\hline 40. & $\begin{array}{l}\text { Arnebia euchroma (Royle ex Benth.) I. M. } \\
\text { Johnston }\end{array}$ & 0 & 0 & 0 & 13.53 & 0 \\
\hline 41. & Arnebia griffithii Boiss., Diagn. & 0 & 0 & 0 & 12.12 & 0 \\
\hline 42. & Arnebia hispidisma (Lehm.) A. DC & 0 & 0 & 0 & 14.77 & 0 \\
\hline 43. & Artemisia parviflora Roxb ex. D. Don & 0 & 0 & 0 & 22.76 & 0 \\
\hline 44. & Artemisia persica Boiss, Diagn. & 0 & 0 & 0 & 17.47 & 0 \\
\hline 45. & $\begin{array}{l}\text { Astragalus coluteocarpus Boiss. ssp. } \\
\text { chitralensis Wenninger, Mitt. Bot. }\end{array}$ & 0 & 0 & 0 & 0 & 17.76 \\
\hline 46. & Astragalus minuto-foliolatus Wendelbo & 0 & 0 & 0 & 0 & 11.79 \\
\hline 47. & Asyneuma strictum Wendelbo. & 0 & 0 & 0 & 10.47 & 0 \\
\hline 48. & Acantholimon stocksii Boiss. & 14.53 & 8.87 & 13.84 & 0 & 0 \\
\hline
\end{tabular}




\begin{tabular}{|c|c|c|c|c|c|c|}
\hline S.No & Plant Species & Site I & Site II & Site III & Site IV & Site V \\
\hline 49. & Acantholimon longiscapum Bokhari & 0 & 20.98 & 0 & 0 & 0 \\
\hline 50. & $\begin{array}{c}\text { Achillea millefolium subsp. } \\
\text { chitralensis }\end{array}$ & 9.47 & 0 & 0 & 0 & 0 \\
\hline 51. & Adonis aestivalis L. & 10.26 & 0 & 0 & 0 & 0 \\
\hline 52. & Agrostis nervosa Nees ex Trin. & 9.06 & 13.09 & 0 & 0 & 0 \\
\hline 53. & Agrostis viridis Gouan, Hort. & 0 & 11.41 & 0 & 0 & 0 \\
\hline 54. & Alcea nudiflora (Lindl.) Boiss. & 11.63 & 13.60 & 0 & 0 & 0 \\
\hline 55. & $\begin{array}{c}\text { Anemone rupicola var. sericea Hook.f.\& } \\
\text { Thomson }\end{array}$ & 10.08 & 13.14 & 0 & 0 & 0 \\
\hline 56. & Anthemis cotula $\mathrm{L}$ & 9.66 & 11.81 & 0 & 0 & 0 \\
\hline 57. & Artemisia scoparia Waldst.\& Kit. & 10.49 & 0 & 0 & 0 & 0 \\
\hline 58. & Artemisia sieversiana Ehrh. & 17.11 & 0 & 0 & 0 & 0 \\
\hline 59. & Askellia flexuosa (Ledb.) W.A. Weber. & 11.46 & 0 & 0 & 0 & 0 \\
\hline 60. & Aster flaccidus Bunge. & 8.047 & 0 & 0 & 0 & 0 \\
\hline 61. & Astragalus affghanus Boiss. & 0 & 0 & 23.43 & 0 & 0 \\
\hline 62. & Astragalus amberstianus Bth.ex. Royle. & 0 & 0 & 19.69 & 0 & 0 \\
\hline 63. & Astragalus imitensis Ali. & 13.69 & 0 & 0 & 0 & 0 \\
\hline 64. & Astragalus edelbergianus Sirj \& Rech.f. & 0 & 0 & 21.64 & 0 & 0 \\
\hline 65. & Asyneuma strictum Wendelbo. & 0 & 0 & 11.74 & 0 & 0 \\
\hline 66. & Atriplex schugnanica Iljin. & 7.46 & 0 & 0 & 0 & 0 \\
\hline 67. & Anaphalis stantonii Y. Nasir. & 0 & 0 & 0 & 0 & 29.48 \\
\hline 68. & Artemisia brevifolia Wall ex DC. & 0 & 0 & 0 & 0 & 16.64 \\
\hline 69. & Artemisia elegantissim Pamp., Nuovo Giorn. & 0 & 0 & 0 & 0 & 18.44 \\
\hline 70. & Asplenium septentrionale (L.) Hoffm. & 0 & 0 & 0 & 0 & 18.56 \\
\hline 71. & Bromus japonicus Thunb. ex Murr., Syst. & 0 & 0 & 0 & 0 & 10.9 \\
\hline 72. & Brachypodium distachyon (L.) P. Beauv. & 0 & 12.77 & 0 & 0 & 0 \\
\hline 73. & Brachypodium sylvaticum (Huds.) P. Beauv. & 0 & 0 & 9.16 & 0 & 0 \\
\hline 74. & Brassica campestris $\mathrm{L}$. & 16.95 & 0 & 0 & 0 & 0 \\
\hline 75. & Bromus oxyodon Schrenk. & 0 & 0 & 0 & 12.93 & 0 \\
\hline 76. & Bunium persicum (Boiss.) Fedtsch. Rastit & 0 & 6.66 & 0 & 0 & 0 \\
\hline 77. & $\begin{array}{c}\text { Brachyactis roylei (Candolle) } \\
\text { Wendelbo. }\end{array}$ & 0 & 12.21 & 0 & 0 & 0 \\
\hline 78. & Bromus danthoniae Trin. & 11.65 & 0 & 0 & 0 & 0 \\
\hline 79. & Bromus persicus Boiss. & 0 & 10.17 & 0 & 0 & 0 \\
\hline 80. & Bromus ramosus Huds. & 0 & 11.22 & 9.93 & 0 & 0 \\
\hline 81. & Bromus tectorum $\mathrm{L}$. & 10.26 & 0 & 0 & 0 & 0 \\
\hline 82. & Bupleurum kohistanicum E. Nasir & 0 & 0 & 11.20 & 0 & 0 \\
\hline 83. & Calendula officinalis $\mathrm{L}$. & 0 & 6.84 & 0 & 0 & 0 \\
\hline 84. & Calamagrostis decora Hook.f. & 0 & 0 & 0 & 0 & 13.66 \\
\hline 85. & Crepis multicaulis Ledeb.var. congsta & 0 & 0 & 0 & 0 & 12.91 \\
\hline 86. & Cystopteris fragilis (L.) Bernh. & 0 & 0 & 0 & 0 & 16.33 \\
\hline 87. & Capsella bursa-pestoris L. & 18.44 & 0 & 0 & 0 & 0 \\
\hline 88. & Cardaria draba (L.) Desv & 0 & 0 & 10.20 & 0 & 0 \\
\hline 89. & Carex stenocarpa Turcz.ex V. Krecz & 0 & 0 & 13.50 & 0 & 0 \\
\hline 90. & Cerastium cerastioides (L.) Britton. & 0 & 0 & 0 & 10.76 & 0 \\
\hline 91. & Chenopodium botrys L. & 0 & 9.17 & 0 & 0 & 0 \\
\hline 92. & Chenopodium murale $\mathrm{L}$. & 16.72 & 0 & 0 & 0 & 0 \\
\hline 93. & Chesneya cuneata (Benth.) Ali. & 0 & 0 & 0 & 0 & 9.68 \\
\hline 94. & Cirsium griffithii Boiss. & 0 & 0 & 0 & 11.38 & 11.93 \\
\hline 95. & $\begin{array}{c}\text { Cirsium wallichii var. glabratum (Hook. f.) } \\
\text { Wendelbo }\end{array}$ & 0 & 0 & 13.52 & 0 & 0 \\
\hline 96. & $\begin{array}{c}\text { Clematis alpina var. sibirica (L.) O. Kuntze, } \\
\text { Verh. }\end{array}$ & 0 & 13.32 & 0 & 0 & 0 \\
\hline 97. & Convulvulus arvensis $\mathrm{L}$. & 9.83 & 0 & 0 & 0 & 0 \\
\hline 98. & Conyza aegyptiaca (L.) Dryand. ex Aiton & 16.38 & 0 & 0 & 0 & 0 \\
\hline 99. & Conyza canadensis (L.) Cronquist. & 0 & 8.30 & 7.44 & 0 & 0 \\
\hline
\end{tabular}




\begin{tabular}{|c|c|c|c|c|c|c|}
\hline S.No & Plant Species & Site I & Site II & Site III & Site IV & Site V \\
\hline 100. & Coriandrum stivum $\mathrm{L}$. & 0 & 9.57 & 10.26 & 0 & 0 \\
\hline 101. & Coronopus didymus (L.) Sm. & 14.26 & 0 & 0 & 0 & 0 \\
\hline 102. & Crepis aitchisonii Boiss. & 0 & 0 & 0 & 10.24 & 0 \\
\hline 103. & Crepis multicaulis Ledeb. var. congsta & 0 & 0 & 0 & 0 & 7.46 \\
\hline 104. & Cuscuta villosa $\mathrm{L}$. & 0 & 0 & 0 & 9.79 & 0 \\
\hline 105. & Cynodon dactylon (L.) Pers. & 18.14 & 0 & 0 & 0 & 0 \\
\hline 106. & $\begin{array}{l}\text { Calamagrostis pseudophragmites subsp. } \\
\text { speudophragmites (Hall.f.) Koel. }\end{array}$ & 0 & 0 & 13.05 & 0 & 0 \\
\hline 107. & Capparis spinosa L. & 15.33 & 14.4 & 0 & 0 & 0 \\
\hline 108. & Chenopodium foliosum (Merrich.) Aschers. & 8.04 & 0 & 0 & 0 & 0 \\
\hline 109. & Cichoriun intybus $\mathrm{L}$. & 11.87 & 0 & 0 & 0 & 0 \\
\hline 110. & Cirsium arvense (L.) Scop. & 8.66 & 15.23 & 14.90 & 0 & 0 \\
\hline 111. & $\begin{array}{c}\text { Codonopsis clematidea (Schrenk) C.B. } \\
\text { Clarke. }\end{array}$ & 8.26 & 0 & 0 & 0 & 0 \\
\hline 112. & Dicanthium annulatum Forssk. Stapf. & 0 & 0 & 9.98 & 0 & 0 \\
\hline 113. & Dactylorhiza umbrosa (Kar. \& Kir.) Nevski. & 0 & 0 & 0 & 12.93 & 0 \\
\hline 114. & $\begin{array}{c}\text { Draba tibetica var. chitralensis (O. E. Nasir) } \\
\text { Jafri. }\end{array}$ & 0 & 0 & 0 & 10.21 & 0 \\
\hline 115. & Equisetum ramossimum Desf. & 0 & 7.20 & 8.59 & 0 & 0 \\
\hline 116. & Ephedra gerardiana Wall.ex Stapf. & 0 & 0 & 15.87 & 0 & 0 \\
\hline 117. & $\begin{array}{l}\text { Eremurus stenophyllus subsp. stenophyllus S. } \\
\text { I. Ali }\end{array}$ & 0 & 0 & 21.04 & 0 & 0 \\
\hline 118. & Ferula narthex Boiss. & 19.5 & 0 & 0 & 0 & 0 \\
\hline 119. & Ferula jaeschkeana Vatke. & 0 & 0 & 0 & 11.29 & 0 \\
\hline 120. & Ferula hindukushensis Kitamura. & 0 & 0 & 0 & 0 & 17.71 \\
\hline 121. & Festuca olgae (Regel) Krivot. & 0 & 0 & 0 & 0 & 12.16 \\
\hline 122. & $\begin{array}{c}\text { Fragaria nubicola (Hook.f.) Lindl.ex } \\
\text { Lacaita }\end{array}$ & 0 & 0 & 10.37 & 0 & 0 \\
\hline 123. & $\begin{array}{l}\text { Glycyrrhiza glabra var. glandulifera } \\
\text { (Waldst. \& Kit.) Boiss. }\end{array}$ & 0 & 0 & 9.68 & 0 & 0 \\
\hline 124. & Heracleum polyadenum Rech.f. \& Riedl. & 0 & 0 & 0 & 0 & 12.16 \\
\hline 125. & Juniperus excelsa M. Bieb & 0 & 0 & 19.69 & 0 & 0 \\
\hline 126. & Kobresia laxa Nees, Contr. & 0 & 0 & 0 & 0 & 22.75 \\
\hline 127. & Koelpinia linearis Pall. var. linearis & 0 & 0 & 0 & 0 & 15.58 \\
\hline 128. & Lactuca serriola $\mathrm{L}$. & 11.89 & 7.57 & 0 & 0 & 0 \\
\hline 129. & Lagochilus cabulicus Bth. & 0 & 0 & 0 & 0 & 11.26 \\
\hline 130. & Lindelofia anchusoides (Lindl.) Lehm. & 0 & 0 & 8.53 & 0 & 0 \\
\hline 131. & Lolium temulentum $\mathrm{L}$. & 0 & 0 & 9.6 & 0 & 0 \\
\hline 132. & $\begin{array}{c}\text { Malcolmia cabulica var. topppinii (O.E. } \\
\text { Schulz) Nasir }\end{array}$ & 0 & 0 & 8.5 & 0 & 0 \\
\hline 133. & Myricatis wallichii Less. & 0 & 0 & 0 & 0 & 7.26 \\
\hline 134. & Matthiola flavida Boiss. & 0 & 0 & 8.59 & 0 & 0 \\
\hline 135. & Melica persica Kunth, Rev. Gram. & 0 & 0 & 0 & 10.04 & 0 \\
\hline 136. & Melilotus officinalis (L.) Pall., Reise. & 0 & 9.17 & 0 & 0 & 0 \\
\hline 137. & Mentha longifolia (L.) Huds. & 14.88 & 9.03 & 0 & 0 & 0 \\
\hline 138. & Myosotis avensis (L.) Hill. & 0 & 0 & 0 & 9.39 & 13.28 \\
\hline 139. & Oxyria digyna (L.) Hill, Hort. & 0 & 0 & 0 & 0 & 21.35 \\
\hline 140. & Oxytropis chitralensis Ali. & 0 & 0 & 0 & 0 & 20.96 \\
\hline 141. & Pennisetum flaccidum Griseb & 12.37 & 6.66 & 0 & 0 & 0 \\
\hline 142. & Poa bulbosa $\mathrm{L}$. & 0 & 0 & 0 & 18.20 & 0 \\
\hline 143. & Poa pratensis subsp. pratensis & 0 & 0 & 13.62 & 0 & 0 \\
\hline 144. & Polygonatum geminiflorum Decne. & 8.49 & 7.57 & 9.34 & 0 & 0 \\
\hline 145. & $\begin{array}{c}\text { Primula macrophylla var. macrophylla D. } \\
\text { Don }\end{array}$ & 0 & 0 & 0 & 11.96 & 8.25 \\
\hline 146. & $\begin{array}{l}\text { Pseudognaphalium luteo-album (L.), O. M. } \\
\text { Hilliard \& B. L Burtt }\end{array}$ & 0 & 0 & 0 & 14.52 & 0 \\
\hline 147. & Pseudomertensia chitralensis (Riedl) Riedl & 0 & 0 & 0 & 0 & 13.39 \\
\hline
\end{tabular}




\begin{tabular}{|c|c|c|c|c|c|c|}
\hline S.No & Plant Species & Site I & Site II & Site III & Site IV & Site V \\
\hline 148. & Psoralea drupaceae Bunge. & 0 & 0 & 6.76 & 0 & 0 \\
\hline 149. & Prangos pabularia Lindl. & 0 & 24.82 & 10.58 & 0 & 0 \\
\hline 150. & $\begin{array}{c}\text { Piptatherum laterale (Munro ex Regel) } \\
\text { Rozhev }\end{array}$ & 0 & 0 & 0 & 0 & 13.66 \\
\hline 151. & Poa alpina $\mathrm{L}$. & 0 & 0 & 0 & 0 & 9.49 \\
\hline 152. & Psychrogeton chitralicus Grierson. & 0 & 0 & 0 & 0 & 16.13 \\
\hline 153. & Puccinellia minuta Bor. & 0 & 0 & 0 & 0 & 20.25 \\
\hline 154. & $\begin{array}{c}\text { Ranunculus laetus Wall.ex Hook.f. \& } \\
\text { Thoms. }\end{array}$ & 0 & 11.22 & 12.37 & 0 & 0 \\
\hline 155. & Ribes orientale Desf. & 0 & 21.79 & 12.34 & 0 & 0 \\
\hline 156. & Rheum webbianum Royle. & 0 & 0 & 0 & 13.67 & 0 \\
\hline 157. & $\begin{array}{c}\text { Rhodiola heterodonta (Hook.f. \& Thomson) } \\
\text { Boriss. }\end{array}$ & 0 & 0 & 0 & 9.47 & 0 \\
\hline 158. & Rhodiola wallichiana (Hook.) S.H. Fu & 0 & 0 & 0 & 22.0 & 0 \\
\hline 159. & Rorippa islandica (Oeder) Borbas. & 0 & 0 & 0 & 9.20 & 0 \\
\hline 160. & Rosularia adenotricha subsp. adenotricha & 0 & 0 & 0 & 9.82 & 0 \\
\hline 161. & Rosularia alpestris (Kar \& Kir) Boiss. & 0 & 0 & 0 & 22.06 & 8.06 \\
\hline 162. & Rubia tibetica Hook.f. & 0 & 0 & 0 & 16.34 & 0 \\
\hline 163. & Rumex hastatus D. Don & 10.37 & 0 & 0 & 0 & 0 \\
\hline 164. & Saussurea leptophylla Hemsl. & 0 & 0 & 0 & 11.03 & 0 \\
\hline 165. & Scandix pecten-veneris L. & 0 & 7.75 & 0 & 0 & 0 \\
\hline 166. & Scutellaria multicaulis Boiss. & 0 & 0 & 0 & 20.79 & 0 \\
\hline 167. & $\begin{array}{c}\text { Seriphidium brevifolium (Wall. ex DC.) } \\
\text { Ling \& Y. R. Ling }\end{array}$ & 0 & 0 & 0 & 17.79 & 0 \\
\hline 168. & $\begin{array}{l}\text { Seriphidium chitralense } \\
\text { (Podlech)Y. R. Ling }\end{array}$ & 0 & 0 & 0 & 13.17 & 14.18 \\
\hline 169. & Silene conoidea $\mathrm{L}$. & 0 & 8.84 & 0 & 0 & 0 \\
\hline 170. & Silene vulgaris (Moench) Garcke. & 0 & 0 & 9.22 & 0 & 0 \\
\hline 171. & Sisymbrium brassiciforme C. A. Мey. & 0 & 0 & 8.53 & 0 & 0 \\
\hline 172. & Solanum nigrum $\mathrm{L}$. & 0 & 6.84 & 0 & 0 & 0 \\
\hline 173. & Solenanthus circinnatus Ledeb. & 0 & 0 & 0 & 9.11 & 0 \\
\hline 174. & Sonchus asper (L.) Hill. & 0 & 0 & 7.93 & 0 & 0 \\
\hline 175. & Stellaria decumbens Edgew. & 0 & 0 & 5.2 & 0 & 0 \\
\hline 176. & Stellaria media (L.) Vill. & 0 & 10.67 & 0 & 0 & 0 \\
\hline 177. & Stipa chitralensis Bor. & 0 & 8.99 & 0 & 0 & 0 \\
\hline 178. & Tanacetum chitralense (Podlech) K. & 0 & 9.67 & 0 & 0 & 0 \\
\hline 179. & Tetrapogon villosus Desf. & 0 & 16.0 & 0 & 0 & 0 \\
\hline 180. & Thlaspi perfoliatum $\mathrm{L}$. & 0 & 13.1 & 0 & 0 & 0 \\
\hline 181. & Trisetaria loeflingiana (L.) Paunero & 0 & 14.3 & 0 & 0 & 0 \\
\hline 182. & $\begin{array}{c}\text { Tanacetum griffithii (C. B. Clarke) } \\
\text { Muradyan. }\end{array}$ & 0 & 0 & 0 & 0 & 19.95 \\
\hline 183. & $\begin{array}{c}\text { Taraxacum brevirostre Hand-Mazz. var. } \\
\text { lanatum. }\end{array}$ & 0 & 0 & 0 & 13.49 & 0 \\
\hline 184. & Taraxacum chitralense Soest. & 0 & 0 & 0 & 0 & 13.36 \\
\hline 185. & Taraxacum officinale Weber. & 0 & 9.57 & 6.13 & 0 & 0 \\
\hline 186. & Taraxacum tricolor V.S & 0 & 0 & 0 & 15.88 & 8.82 \\
\hline 187. & Taraxacum wendelboanum Soest. & 0 & 0 & 0 & 0 & 9.65 \\
\hline 188. & Taraxacum obtusum (Soest) R. Doll & 0 & 0 & 0 & 0 & 8.74 \\
\hline 189. & Tragopogon gracilis D. Don. & 0 & 0 & 0 & 10.08 & 0 \\
\hline 190. & Tricholepis toppinii Dunn. & 0 & 0 & 0 & 14.90 & 16.37 \\
\hline 191. & Tussilago farfara $\mathrm{L}$. & 0 & 0 & 0 & 7.75 & 0 \\
\hline 192. & Verbascum thapsus L. & 0 & 0 & 9.06 & 0 & 0 \\
\hline 193. & Verbena officinalis $\mathrm{L}$. & 0 & 8.30 & 5.5 & 0 & 0 \\
\hline 194. & Xanthium strumarium L. & 0 & 8.627 & 7.44 & 0 & 0 \\
\hline 195. & Youngia japonica (L.) DC. & 0 & 12.14 & 0 & 0 & 0 \\
\hline
\end{tabular}




\section{A. Community structure of foothills vegetation}

\section{Shagrom (Site-I)}

\section{Elaeganus-Prunus-Adiantum community (EPA)}

This plant community was established at Shagrom at an elevation of $1000 \mathrm{~m}-1550 \mathrm{~m}$ having 21 species. Elaeganus angustifolia (IV. 24.31), Prunus jacquemontii (IV. 19.99), and Adiantum venustum (IV. 19.66) were the dominant plant species. Co-dominant species were the Berberis lyceum, Capsella bursa-pestoris, Brassica campestris, Conyza aegyptiaca, Cynodon dactylon and Mentha longifolia (Table 2). Soil of this site was basic in nature having $\mathrm{pH} 8.5$ and electrical conductivity $0.4 \mathrm{dsm}^{-1}$. Similarly soil texture has been reported with $2.6 \%$ clay, $38.0 \%$ silt and $58.86 \%$ sand, respectively. Organic matter was $2.05 \%$ followed by calcium carbonate $13 \%$. The nitrogen content $0.1035 \%$, phosphorus $15.28 \mathrm{mg} / \mathrm{kg}$ and potassium $114.33 \mathrm{mg} / \mathrm{kg}$ was recorded respectively (Table 3).

Table 3. Edaphic variables, mineral content and plant communities at 5 monitoring sites

\begin{tabular}{c|c|c|c|c|c|c|c|c|c|c}
\hline \multirow{2}{*}{ Parameters } & \multicolumn{3}{|c|}{ Foothills } & \multicolumn{3}{c|}{ Midhills } & \multicolumn{3}{c}{ Sub-alpine \& Alpine } \\
\cline { 2 - 10 } & S & W & Z & S* & W* & $\mathbf{Z}^{*}$ & RGN & RGS & GN & GS \\
\hline Clay \% & 2.66 & 4.56 & 20.61 & 5.67 & 0.81 & 11.6 & 6.51 & 6.3 & 2.79 & 2.91 \\
Silt \% & 38 & 30.66 & 38.01 & 42 & 18.01 & 15 & 24.99 & 25.9 & 34.2 & 30.20 \\
Sand \% & 58.86 & 62.86 & 39.50 & 51.39 & 76.41 & 70.4 & 72.21 & 70.2 & 61.21 & 61.2 \\
\hline Texture class & \multicolumn{10}{|c}{ Sandy loam } \\
\hline pH & 8.5 & 6.63 & 8.1 & 6.67 & 6.39 & 8.1 & 6.6 & 6.6 & 7.8 & 7.5 \\
O-M \% & 2.05 & 2.16 & 2.28 & 2.1 & 1.87 & 1.55 & 0.81 & 0.84 & 0.75 & 0.78 \\
Lime \% & 13 & 16.25 & 8.4 & 5.01 & 5.49 & 11.4 & 0.51 & 0.51 & 2.7 & 2.49 \\
Ec x10 dsm ${ }^{-1}$ & 0.4 & 0.06 & 0.29 & 0.12 & 0.20 & 0.21 & 0.48 & 0.48 & 0.26 & 0.36 \\
N \% & 0.10 & 0.11 & 0.11 & 0.10 & 0.08 & 0.08 & 0.12 & 0.14 & 0.12 & 0.15 \\
P (mg/kg) & 15.28 & 5.87 & 12.16 & 20.39 & 55.67 & 12.2 & 14.01 & 15.9 & 12 & 15.99 \\
K (mg/kg) & 114.3 & 96.33 & 187 & 116 & 238 & 132 & 183.8 & 186 & 84.2 & 88.2 \\
\hline
\end{tabular}

Key: S-Shagrom, W-Warimon, Z-Zondrangam, $\mathbf{S}^{*}$-Shagrom, $\mathbf{W}^{*}$-Warimon, $\mathbf{Z}^{*}$-Zondrangam, RGNRosh Gol Northern slope, RGS-Rosh Gol Southern slope, GN-Ghari Northern slope, GS-Ghari Southern slope

\section{Warimon (Site-II)}

\section{Fraxinus-Rosa-Acanthophyllum community (FRA)}

This community was established at an altitude of $1200 \mathrm{~m}-1600 \mathrm{~m}$ comprised of 30 species. Dominant species of this community includes Fraxinus xanthxyloides (IV. 19.63), Rosa webbiana (IV. 17.93) and Acanthophyllum laxiflorum (IV. 17.92). Co-dominant shrub species include Crataegus songarica, Tamaricaria elegans, Rhamnus prostrata, Clematis alpina, and Cotoneaster affinis who grew around this community (Table 2). Soil $\mathrm{pH}$ was 6.6 and electrical conductivity $0.06 \mathrm{dsm}^{-1}$. The soil consisted of $4.56 \%$ clay, $30.66 \%$ silt and $62.86 \%$ sand, textured with sandy loam. Soil contained $2.16 \%$ organic matter and $16.25 \%$ calcium carbonate. Nitrogen content was $0.114 \%$ which was better than Shagrom community. Phosphorus content was 5.87 
$\mathrm{mg} / \mathrm{kg}$ and potassium $96.33 \mathrm{mg} / \mathrm{kg}$ was also estimated which has a normal range required for the plant growth (Table 3).

Zondrangam (Site-III)

Hippophae-Sophora-Poa community (HSP)

This community was established at altitude of $1320 \mathrm{~m}-1760 \mathrm{~m}$ having 32 species. The dominant species of this community was Hippophae rhamnoides (IV. 17.36), Sophora mollis (IV. 13.86) and Poa pratensis (IV. 13.62). The co-dominant species were Carex stenocarpa, Cirsium willichi, Cardaria draba, Coriandrum sativum, Lolium temulentum, and Tamaricaria elegans (Table 2). Soil was sandy loam of this site with $20.61 \%$ clay, $38.01 \%$ silt, and $39.501 \%$ sand. The $\mathrm{pH}$ was 8.1 and $0.291 \mathrm{dsm}^{-1}$ electrical conductivity was also calculated. Similarly, organic matter $2.28 \%$ and calcium carbonate was estimated $8.4 \%$. Nitrogen content was recorded $0.117 \%$ followed by phosphorus $12.16 \mathrm{mg} / \mathrm{kg}$ and potassium $187 \mathrm{mg} / \mathrm{kg}$, respectively (Table 3).

\section{B. Community structure of midhills vegetation}

Shagrom (Site-I)

Ferula-Artemisia-Capparis community (FAC)

This community was composed of 26 species located at an altitude of $2250 \mathrm{~m}-2500 \mathrm{~m}$. Ferula narthex (IV. 19.55), Artemisia sieveriana (IV. 17.11), and Capparis spinosa (IV. 15.33) were the dominant species. The co-dominant species of this community were Cotoneaster racemiflorus, Juniperus communis, Cichorium intybus, Acantholimon stocksii, and Astragalus imitensis (Table 2). The soil $\mathrm{pH}$ was 6.6 and electrical conductivity 0.129 $\mathrm{dsm}^{-1}$ which represents acidic nature of soil. The soil texture was sandy loam and composed of clay $5.67 \%$, silt $42.0 \%$ and sand $51.39 \%$. Organic matter was estimated $2.1 \%$ and calcium carbonate $5.01 \%$. The concentration of nitrogen was calculated $0.104 \%$, phosphorus $20.391 \mathrm{mg} / \mathrm{kg}$ and $116 \mathrm{mg} / \mathrm{kg}$ potassium, respectively (Table 3).

Warimon (Site-II)

Prangos-Ribes-Berberis-community (PRB)

Along $2320 \mathrm{~m}-2420 \mathrm{~m}$ altitude Prangos-Ribes-Berberis-community was recorded having 22 species. Prangos pabularia (IV. 24.82), Ribes orientale (IV. 21.79) and Berberis calliobotrys (IV. 20.74) were dominant species. The co-dominant species of this community includes Tetrapogon villosus, Trisetaria loeflingiana, Thlaspi perfoliatum and Cirsium arvensis (Table 2). The texture of the soil recorded sandy loamy which contains clay $0.681 \%$, silt $18.01 \%$ and sand $76.41 \%$. The $\mathrm{pH}$ of the soil was 6.3 and electrical conductivity $0.201 \mathrm{dsm}^{-1}$. Chemical analysis revealed $1.87 \%$ organic matter and $5.49 \%$ calcium carbonate. The nitrogen concentration was estimated $0.087 \%$ followed by $55.67 \mathrm{mg} / \mathrm{kg}$ phosphorus and $238 \mathrm{mg} / \mathrm{kg}$ potassium (Table 3).

\section{Zondrangam (Site-III)}

\section{Astragalus*-Astragalus-Eremurus community (AAE)}

This community consisted of 20 species which was reported along 2260 m-2350 m altitude. The dominant species were Astragalus affghanus (IV. 23.43), Astragalus edelbergianus (IV. 21.64) and Eremurus stenophyllus (IV. 21.04). Juniperus excelsa, 
Astragalus amberstianus, Acantholimon longiscapum, and Ephedera gerardiana were co-dominants (Table 2). Chemical analysis of soil revealed $1.551 \%$ organic matter, $11.49 \%$ calcium carbonate, $0.081 \%$ nitrogen, $12.24 \mathrm{mg} / \mathrm{kg}$ phosphorus, $132 \mathrm{mg} / \mathrm{kg}$ potassium, $\mathrm{pH} 8.1$ and $0.021 \mathrm{dsm}^{-1}$ electrical conductivity. The soil texture represents sandy loam contributed clay $11.67 \%$, silt $15.0 \%$ and sand $70.41 \%$ (Table 3).

\section{Community structure of sub-alpine and alpine vegetation}

Rosh Gol (Site-IV)

Northern slope

Artemisia-Rhodiola-Rosularia community (ARR)

Artemisia-Rhodiola-Rosularia community was recorded from Rosh Gol along (South facing) of sub-alpine region at an altitude of $3125 \mathrm{~m}-3440 \mathrm{~m}$. This community was consisted of at had 23 species. The dominant species were Artemisia parviflora (IV. 22.76), Rhodiola wallichiana (IV. 22.03) and Rosularia alpestris (IV. 22.06). Seriphidium brevifolium, Rosa ecae, Pseudognaphalium luteo-album, Artemisia persica, and poa bulbosa were co-dominants (Table 2). Texture of the soil was sandy loam with clay $6.51 \%$, silt $24.99 \%$ and sand $72.21 \%$. The soil of this site had $0.81 \%$ organic matter and $0.51 \%$ calcium carbonate. Amount of nitrogen $0.12 \%$, phosphorus $14.01 \mathrm{mg} / \mathrm{kg}$ and potassium $183.8 \mathrm{mg} / \mathrm{kg}$ was estimated. The $\mathrm{pH}$ was calculated 6.6 and electrical conductivity $0.489 \mathrm{dsm}^{-1}$ (Table 3 ).

\section{Southern slope}

\section{Betula-Scutularia-Taraxicum community (BST)}

This community consisted of 21 species at Rosh Gol along (North facing) of the sub-alpine region at an altitude of $3320 \mathrm{~m}-3650 \mathrm{~m}$. The dominant species were Betula chitralica (IV. 27.21), Scutellaria multicaulis (IV. 20.79) and Taraxacum tricolor (IV. 15.88). The co-dominant species were Tricholepis toppinii, Taraxacum brevirostre, Seriphidium chitralensis, and Arnebia hispidisma (Table 2). The $\mathrm{pH}$ of soil was 6.6 and electrical conductivity $0.48 \mathrm{dsm}^{-1}$. The soil was sandy loam with clay $6.3 \%$, silt $25.94 \%$ and sand $70.2 \%$. Chemical properties of the soil showed organic matter $0.84 \%$ and calcium carbonate $0.51 \%$, respectively. The nitrogen concentration $0.141 \%$, phosphorus $15.99 \mathrm{mg} / \mathrm{kg}$ and potassium $186.8 \mathrm{mg} / \mathrm{kg}$ was investigated (Table 3).

\section{Ghari (Site-V)}

Northern slope

\section{Anaphalis-Cousina-Kobresia community (ACK)}

This community had 19 species at Ghari site of the alpine zone (South facing) located at an elevation of 2870 m-2950 m. Anaphalis stantonii (IV. 29.48), Cousinia pycnoloba (IV. 25.74) and Kobresia laxa (IV. 22.25) were dominants; while Puccinellia minuta, Psychrogeton chitralicus, Cystopteris fragilis, Ferula hindukushhensis, Koelpinia linearis, and Asplenium septentrionale were co-dominants (Table 2). Soil of this site had sandy loam texture with clay $2.79 \%$, silt $34.2 \%$ and sand $61.2 \%$. The $\mathrm{pH}$ was calculated 7.89 and electrical conductivity $0.2679 \mathrm{dsm}^{-1}$. Similarly, organic matter $0.75 \%$ and calcium carbonates $2.7 \%$. Nitrogen concentration was recorded $0.12 \%$, followed by phosphorus $12 \mathrm{mg} / \mathrm{kg}$ and potassium $84.2 \mathrm{mg} / \mathrm{kg}$ (Table 3). 


\section{Southern slope}

\section{Alajja-Oxyria-Oxytropis community (AOO)}

Ghari alpine zone (North facing) located at an altitude of $3620 \mathrm{~m}-4200 \mathrm{~m}$. The community of this site had 23 species. The dominant species of this community included Allaja rhomboidea (IV. 24.63), Oxyria digyna (IV. 21.35) and Oxytropis chitralensis (IV. 20.96). Tanacetum grifthii, Alotis smithii, Astragalus coluteocarpus, and Betula utilis were co-dominants (Table 2). Soil of this site was sandy loam in texture with $2.91 \%$ clay, $30.201 \%$ silt and $61.2 \%$ sand particles. Soil $\mathrm{pH}$ was 7.5 and organic matter content was estimated $0.78 \%$. Calcium carbonate was found to be $2.49 \%$. Similarly, concentration of nitrogen $0.15 \%$, phosphorus $15.99 \mathrm{mg} / \mathrm{kg}$ and potassium $88.2 \mathrm{mg} / \mathrm{kg}$ was also estimated (Table 3).

\section{Similarity index}

According to Motyka's index of similarity (Motyka et al., 1950), FRA community was found to be $46.1 \%$ similar to HSP community. The similarity between PRB community and $\mathrm{A}^{*} \mathrm{AE}$ community was to be found $28.5 \%$. The analysis showed that similarity between EPA community and that of FRA community was $23.52 \%$. Similarly, index of FAC and PRB communities was $8.33 \%$. EPA community was 7.54\% similar to HSP community. Only $5.12 \%$ similarity was observed between A*AE community and ACK community. Similarity between ACK, EPA communities and ACK, BST communities was recorded $5 \%$ each; followed by similarity of A*AE, EPA communities and ACK, PRB communities $4.87 \%$ each. Similarity index was observed for EPA, ARR, EPA, BST, ACK, ARR and ACK, AOO 4.76\% each respectively. EPA community showed minimum similarity with PRB community i.e. $4.65 \%$, while the remaining communities had less than $4 \%$ similarity index among themselves (Table 4). Seasonal variation was the main cause of similarity index between communities. The high similarity index between the communities is due to characteristics such as similarity in composition of nutrients, the proximity of stands to each other that had almost similar habitat conditions in terms of $\mathrm{N}, \mathrm{P}, \mathrm{K}, \mathrm{pH}$ and soil from clay loam to sandy loam. The high similarity index among some plant communities during the monsoon and spring seasons might be due to the occurrence of the same shrubs, trees, evergreen perennial herbs and geophytes while most of plant communities dominated by annuals showed least similarity among themselves.

\section{Simpson diversity index}

Species diversity was obtained by Hussain (1989) method. Species diversity is affected by various biotic and abiotic factors including elevation, climatic conditions, edaphic characteristics, grazing, browsing, and anthropogenic activities. Species diversity is the main attribute of vegetation dynamics, which reflects the composition and productivity of vegetation. Species diversity calculates the complexity and function within the community. At Site-I Simpson's diversity was found to be 0.1149 followed by Site-II and Site-III with diversity values of 0.159 and 0.1069 , respectively. At SiteIV diversity was 0.1004 followed by Site-V with a value of 0.111 , Site-VI 0.118 , SiteVII 0.127 , Site-VIII 0.110 and Site- X had diversity value of 0.174 . The lowest value was recorded for Site-IX i.e. 0.106 (Table 5, Fig. 2). 
Table 4. Similarity index for 10 different communities

\begin{tabular}{c|c|c|c|c|c|c|c|c|c|c}
\hline Communities & EPA & FRA & HSP & FAC & PRB & AAE & ARR & BST & ACK & AOO \\
\hline EPA & $\mathrm{X}$ & $\mathrm{X}$ & $\mathrm{X}$ & $\mathrm{X}$ & $\mathrm{X}$ & $\mathrm{X}$ & $\mathrm{X}$ & $\mathrm{X}$ & $\mathrm{X}$ & $\mathrm{X}$ \\
FRA & 23.52 & $\mathrm{X}$ & $\mathrm{X}$ & $\mathrm{X}$ & $\mathrm{X}$ & $\mathrm{X}$ & $\mathrm{X}$ & $\mathrm{X}$ & $\mathrm{X}$ & $\mathrm{X}$ \\
$\mathrm{HSP}$ & 7.54 & 46.1 & $\mathrm{X}$ & $\mathrm{X}$ & $\mathrm{X}$ & $\mathrm{X}$ & $\mathrm{X}$ & $\mathrm{X}$ & $\mathrm{X}$ & $\mathrm{X}$ \\
FAC & 4.25 & 3.57 & 3.44 & $\mathrm{X}$ & $\mathrm{X}$ & $\mathrm{X}$ & $\mathrm{X}$ & $\mathrm{X}$ & $\mathrm{X}$ & $\mathrm{X}$ \\
PRB & 4.65 & 3.84 & 3.70 & 8.33 & $\mathrm{X}$ & $\mathrm{X}$ & $\mathrm{X}$ & $\mathrm{X}$ & $\mathrm{X}$ & $\mathrm{X}$ \\
AAE & 4.87 & 4.0 & 3.84 & 4.34 & 28.5 & $\mathrm{X}$ & $\mathrm{X}$ & $\mathrm{X}$ & $\mathrm{X}$ & $\mathrm{X}$ \\
ARR & 4.76 & 3.77 & 3.63 & 4.08 & 4.44 & 4.65 & $\mathrm{X}$ & $\mathrm{X}$ & $\mathrm{X}$ & $\mathrm{X}$ \\
BST & 4.76 & 3.92 & 3.77 & 4.25 & 4.65 & 4.87 & 4.54 & $\mathrm{X}$ & $\mathrm{X}$ & $\mathrm{X}$ \\
ACK & 5.0 & 4.08 & 3.92 & 4.44 & 4.87 & 5.12 & 4.76 & 5.0 & $\mathrm{X}$ & $\mathrm{X}$ \\
AOO & 4.54 & 3.77 & 3.63 & 4.08 & 4.44 & 4.34 & 4.37 & 4.45 & 4.76 & $\mathrm{X}$ \\
\hline
\end{tabular}

Key: EPA-Elaeagnus-Prunus-Adiantum community, FRA-Fraxinus-Rosa-Acanthophylum community, HSP-Hippophae-Sophora-Poa community, FAC-Ferula-Artemisia-Capparis community, PRBPrangos-Ribes-Berberis community, AAE-Astragalus-Astragalus-Eremurus community, ARRArtemisia-Rhodiola-Rosularia community, BST-Betula-Scutellaria-Taraxacum community, ACKAnaphalis-Cousinia-Kobresia community, AOO-Alajja- Oxyria-Oxytropis community

Table 5. Simpson's diversity, among different communities

\begin{tabular}{c|c|c}
\hline Sites & Communities & Simpson's diversity index \\
\hline Site-I & Elaeagnus-Prunus-Adiantum & 0.111 \\
Site-II & Fraxinus-Rosa-Acanthophylum & 0.159 \\
Site-III & Hippophae-Sophora-Poa & 0.1069 \\
Site-IV & Ferula-Artemisia-Capparis & 0.1004 \\
Site-V & Prangos-Ribes-Berberis & 0.111 \\
Site-VI & Astragalus-Astragalus-Eremurus & 0.118 \\
Site-VII & Artemisia-Rhodiola-Rosularia & 0.127 \\
Site-VIII & Betula-Scutellaria-Taraxacum & 0.110 \\
Site-IX & Anaphalis-Cousinia-Kobresia & 0.106 \\
Site-X & Alajja-Oxyria-Oxytropis & 0.174 \\
\hline
\end{tabular}

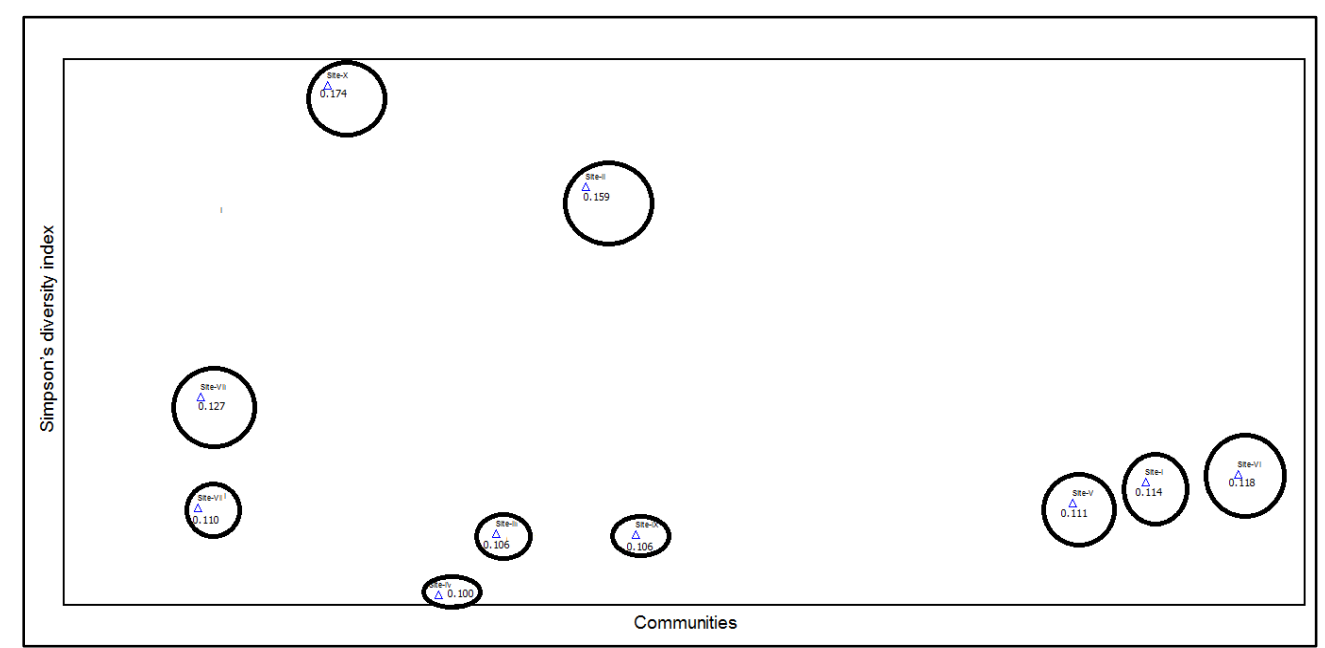

Figure 2. Simpson's diversity, among communities through PC-Ord 


\section{Species richness}

Species richness was calculated after Menhinick (1964). The species richness of a specific region is the result of various environmental factors such as geography, topography, species pool, area productivity and species competition. In the present study the maximum species richness was recorded 0.132 at Rosh Gol North slope followed by 0.104 at Shagrom Midhills, 0.18 at Ghari south slope and 0.16 at Warimon. The high species richness is directly linked with favorable environmental conditions and soil factors. The low species richness 0.12 was recorded in the foothills of Shagrom. Similarly, midhills of Warimon and the north slope of Ghari contributed 0.11 species richness, followed by foothills 0.10 species richness of Zondrangam and south slope of Rosh Gol respectively (Table 6, Fig. 3). Variation in altitude and physicochemical properties of soil may also have profound influence on species richness. The species richness was high during spring but decreased during the monsoon season. This is due to dominance of annuals and geophytes during spring which disappear at the onset of the monsoon season. The low species richness was due to lack of water, high temperature, overgrazing and deforestation. However, in contrast to this, the species richness follows the interpolated richness pattern.

Table 6. Species richness and maturity index among different communities

\begin{tabular}{c|c|c|c}
\hline Sites & Communities & SR & MI \\
\hline Site-I & Elaeagnus-Prunus-Adiantum & 0.12 & 31.42 \\
Site-II & Fraxinus-Rosa-Acanthophylum & 0.16 & 27.00 \\
Site-III & Hippophae-Sophora-Poa & 0.10 & 27.18 \\
Site-IV & Ferula-Artemisia-Capparis & 0.10 & 37.69 \\
Site-V & Prangos-Ribes-Berberis & 0.11 & 31.36 \\
Site-VI & Astragalus-Astragalus-Eremurus & 0.12 & 35.5 \\
Site-VII & Artemisia-Rhodiola-Rosularia & 0.13 & 32.60 \\
Site-VIII & Betula-Scutellaria-Taraxacum & 0.10 & 37.14 \\
Site-IX & Anaphalis-Cousinia-Kobresia & 0.11 & 35.26 \\
Site-X & Alajja-Oxyria-Oxytropis & 0.18 & 31.30 \\
\hline
\end{tabular}

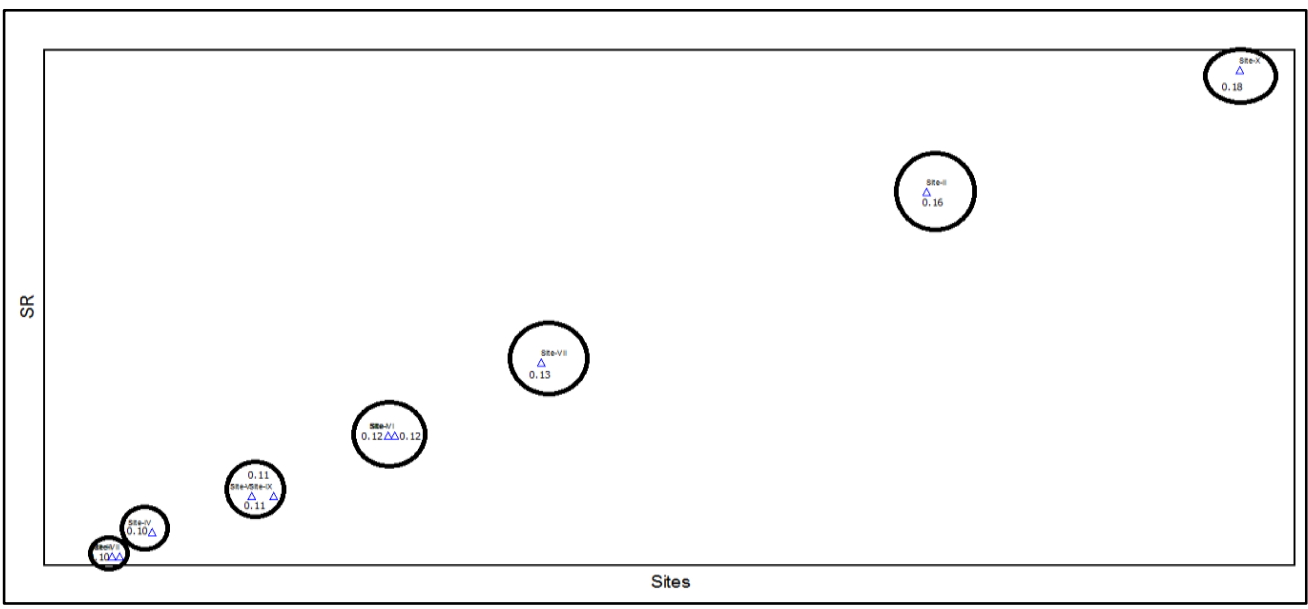

Figure 3. Species richness among communities through PC-Ord 


\section{Maturity index}

Maturity index was calculated according to Pichi-Sermolli (1948). This is a new index for appraising the vegetation pattern of an area affected by environmental and edaphic conditions. During the present study, index of maturity of communities ranged from 27.0 to 37.69 . The highest value of maturity index 37.69 was recorded in the communities of Shagrom. Similarly, maturity index of the communities of south slope Rosh Gol 37.14, followed by the midhills of Zondrangam 35.5. The maturity index of communities in North slope Ghari 35.26 and North slope Rosh Gol was 32.60. Maturity index of the communities of foothills Shagrom was 31.42, while in the midhills communities of Warimon was 31.36. The lowest maturity index 27.18 was reported in south slope communities of Ghari and foothills communities of Warimon 27.0 (Table 6, Fig. 4). All the stands were immature which might be due to lesser adaptation to the microclimate of the study sites. This was further enhanced due to high anthropogenic pressures which disturbed the natural balance of the plant communities and prevented them from attaining maturity. Low maturity reflects the heterogeneity within the plant communities as a result of poor adaptability to the ecological conditions of area.

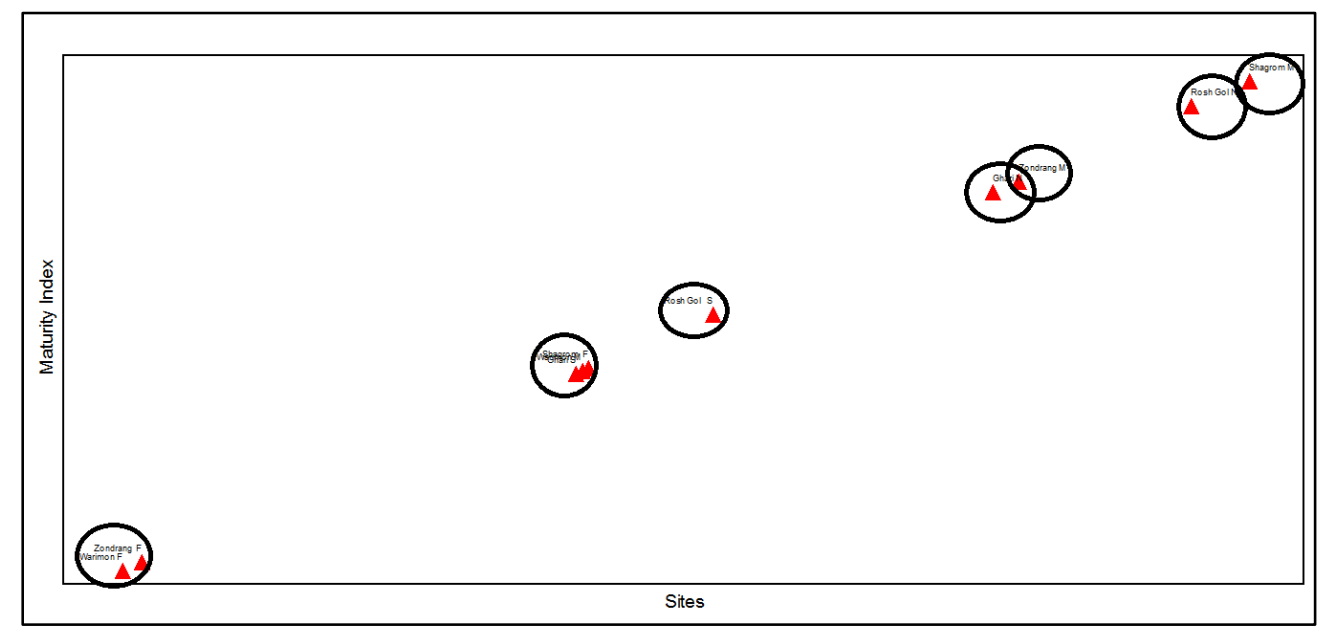

Figure 4. Maturity index among communities through PC-Ord. Key to abbreviations used in Figs. 2 , 3 \& 4: Site-I: Shagrom (Foothills), Site-II: Warimon (Foothills), Site-III: Zondrangam (Foothills), Site-IV: Shagrom (Midhills), Site-V: Warimon (Midhills), Site-VI: Zondrangam (Midhills), Site-VII: Rosh Gol Northern slope, Site-VIII: Rosh Gol Southern slope, Site-IX: Ghari Northern slope, Site-X: Ghari Southern slope

\section{Discussion}

During current study the vegetation of Terich valley, was classified into ten plant communities based on importance values (IVs) which is in line with the works of Hussain et al. (2010, 2019), Ali et al. (2018), Srivastava et al. (2016), Gandhi and Sundarapandian (2014), Altay et al. (2012), Zhang and Zhang (2011), Tsiourlis et al. (2009), Liu (2008), Hirst and Jackson (2007) and Oswalt et al. (2006). At Site-I foothills and midhills EPA-FCA communities were established. Distribution of trees was very rare due to lavish wood cutting in this site. Inadequate trees and relatively thick bushes spread close to the lower regions showed the disturbed nature of habitat which is under gigantic biotic pressure. Expanding population and development of 
residential units is the main reason behind the species loss in this locality. Similar communities are reported from their respective study areas by Ali et al. (2018), Hussain et al. (2015), Mehmood et al. (2015), Ilyas et al. (2015) and Rashid et al. (2011). Site-II foothills and midhills had FRA-PRB communities. Species of Trees and shrubs showed a clumped dispersion pattern at these monitoring sites. Effects of soil disintegration were more visible at Site-I and Site-II where dissolved soils had herbaceous individuals like Conyza aegyptiaca, Cynodon dactylon, Crataegus songarica, Tamaricaria elegans, Rhamnus prostrate and Verbascum thapsus which are the indicator species of eroded soils. Similar communities but with different IV are reported by Hussain et al. (2019), Khan et al. (2016), Ummara et al. (2015), Forzieri et al. (2011) and Wahab et al. (2010). Site-III foothills and midhills exhibited HSP-AAE communities. This site had a uniformly spread thick scrubs of Juniperus, Astragalus, Acantholimon, and Ephedera. Deforestation was likewise not excessively disturbing when contrasted with that of foothills and midhills of Terich valley monitoring sites. At higher altitude the valley was practically undisturbed anyway the environment will confront biotic pressure, particularly the anthropogenic stresses from increasing population in the low laying villages in the next few years. These findings are backed by works of Hussain et al. (2015), Khan et al. (2016), Ali et al. (2015), Ilyas et al. (2015), Sharma et al. (2014), Ali and Malik, (2010) and Wahab et al. (2010). ARR-BST communities were found at north and south slopes of site-IV. This sub-alpine zone supported the richness of Artemisia parviflora, Rhodiola wallichiana, Rosularia alpestris, Seriphidium brevifolium, Rosa ecae, Pseudognaphalium luteo-album, Artemisia persica, and poa bulbosa. This is line with findings of Ali et al. (2018), Ilyas et al. (2015), Siddiqui et al. (2011), Ahmed et al. (2011) and Hussain et al. (2004). At north and south slopes of site-V, ACK-AOO communities were recorded from this alpine zone. Plants of this site was found healthy with the special case on Northern slants where deforestation was very clear because of fuel wood extraction by local people for Betula utilis. These results are in line with the works of Haq et al. (2015), Naveed (2014), Ilyas et al. (2012), Khan et al. (2010, 2011), and Ahmed et al. (2009).

\section{Conclusion}

This research shows vegetation dynamics based on 195 sampling units at 5 monitoring sites. Soil analyses cover 11 parameters with different properties at each site. Variations in edaphic variables, temperature, humidity and slope are due to spatial variations among plant communities at different monitoring sites. Vegetation is endangered in the study region and needs to be conserved until it's too late. The vegetation cover decreases anthropogenic factors, over grazing, natural calamities, and erosion. Extraction of Terich valley trees by local communities, particularly during harsh winters, not only reduces the density of the tree, but also increases the rate of soil erosion. Climate change has not spared vegetation in the Hindukush range and with each passing year, floral diversity is growing. Impacts of anthropogenic pressure for maturity index are evident from the low ratings. Highest similarity index was recorded for community FRA which was found to be $46.1 \%$ similar to HSP community. Highest maturity index value was recorded for communities FAC at Site-IV i.e. 37.69 followed by communities BST at Site-VIII i.e. 37.14. Species richness of community AOO was estimated high 0.18 at Site-X. At Site-X Simpson's diversity was found to be maximum for community AOO 0.17 followed by community FRA at Site-II with diversity values 
of 0.15 , respectively. Low maturity index values suggest the plant communities' inability to cope with anthropogenic, edaphic, and climatic stresses. Likewise, low values of index of similarity indicate the heterogeneity between plant communities. The structure of vegetation indicates local flora is fighting a war of its survival. Once covered under a thick blanket of vegetation, bare patches are now visible on mountain slopes. Proper management of the grazing should be ensured for understory plant regeneration. To check the erosion due to landsliding and flood, fast-growing plant species must be cultivated to rehabilitate the degraded and fragmented habitat. A comprehensive program must be designed through involving local masses, conservationists, Governmental and Non-Governmental Organizations to take useful measures for threatened plants and their conservation with special focus on endemic species to mitigate their extinction risk.

Acknowledgements. This work is a part of Ph.D research of first author.

Conflict of interests. No potential conflict of interests was reported by the authors.

\section{REFERENCES}

[1] Ahmad, S. S. (2009): Ordination and classification of herbaceous vegetation in Margalla Hills National Park Islamabad Pakistan. - Bio. Div. Cons. 2(2): 38-44.

[2] Ahmad, M., Shaukat. S. S. (2012): A Text book of vegetation ecology. - Abrar sons Karachi, Pakistan, pp. 302-305.

[3] Ahmed, M., Hussain, T. A., Sheikh, H. S., Hussain, S., Siddiqui, M. F. (2006): Phytosociology and structure of Himalayan forests from different climatic zones of Pakistan. - Pak. J. Bot. 38(2): 361-383.

[4] Ahmed, M., Khan, N., Wahab, M., Hamza, S., Saddiqui, F. M., Nazim, K., Khan, M. U. (2009): Vegetation structure of Olea ferruginea forests of district Dir Lower of Pakistan. - Pak. J. Bot. 41(6): 2683-2695.

[5] Ahmed, M., Shaukat, S. S., Saddiqui, M. F. (2011): A multivariate analysis of the vegetation of Cedrus deodara forests in Hindukush and Himalayan ranges of Pakistan: evaluating the structure and dynamics. - Turk. J. Bot. 35: 419-438.

[6] Ali, A., Ahmad, N., Habib, G., Amjed, M. (2007): Study the vegetation capacity and physical characteristics of protected and open rangeland in district Dir (Lower). - Sarhad. J. Agric. 23(1): 17-24.

[7] Ali, S. M., Malik, R. N. (2010): Vegetation communities of urban open spaces: green belts and parks in Islamabad city. - Pak. J. Bot. 42(2): 1031-1039.

[8] Ali, S., Perveen, A., Qaiser, M. (2015): Vegetation structure, edaphology and ethnobotany of Mahaban and Malka (District Buner) KPK, Pakistan. - Pak. J. Bot. 47(SI): 15-22.

[9] Ali, A., Badshah, L., Hussain, F. (2018): Vegetation structure and threats to Montane Temperate Ecosystems in Hindukush Range, Swat, Pakistan. - Applied Ecology and Environmental Research 16(4): 4789-4811.

[10] Altay, V., Ozyigit, I. I., Yarc, C. (2012): Plant Communities in Urban Habitats of Istanbul Turkey. - Pak. J. Bot. 44: 177-186.

[11] Arora, V. (2002): Modeling vegetation as a dynamic component in soil-vegetationatmosphere transfer schemes and hydrological models. - Rev. Geophys. 40(2): 3-1-3-26.

[12] Bakkenes, M., Alkemade, J. R. M., Ihle, F., Leemans, R., Latour, J. B. (2002): Assessing effects of forecasted climate change on the diversity and distribution of European higher plants for 2050. - Global Change Biology 8: 390-407.

[13] Black, C. A. (1965): Methods of Soil Analysis. - Agron Inc. Madison Wisconsin, USA. 
[14] Bouyoucos, G. J. (1936): Directions for making mechanical analysis of soils by the Hydrometer Method. - Soil. Sci. 42(3): 225-230.

[15] Brady, N. C. (1990): The nature and properties of soil. 10 ${ }^{\text {th }}$ ed. - Macmillan Publishing Co. New York, NY, 621p.

[16] Forzieri, G., Degetto, M., Righetti, M., Castelli, F., Preti, F. (2011): Satellite multispectral data for improved floodplain roughness modelling. - Journal of hydrology 407(1-4): 4157.

[17] Gandhi, D. S., Sundarapandian, S. M. (2014): Diversity and distribution pattern of understory vegetation in tropical dry forests of Sathanur reserve forest in Eastren Ghats, India. - Int. J. Sci. Nat. 5(3): 452-461.

[18] Haq, F., Ahmad, H., Iqbal, Z. (2015): Vegetation description and phytoclimatic gradients of subtropical forests of Nandiar Khuwar catchment District Battagram. - Pak. J. Bot. 47(4): 1399-1405.

[19] Hirst, C. N., Jackson, D. A. (2007): Reconstructing community relationships, the impact of sampling error, ordination approach and gradient length. - Divers. Distrib. 13(4): 361371.

[20] Hussain, F. (1989): Field \& Laboratory Manual of Plant Ecology. - UGC. Islamabad.

[21] Hussain, F., Murad, A. (2004): Weed communities in the Potato fields of Mastuj disst. Chitral. - Sci. Khyber. 17(2): 201-206.

[22] Hussain, A., Farooq, M. A., Ahmad, M., Akbar, M., Zafar, M. U. (2010): Phytosociology and structure of central Karakoram national park (CKNP) of Northern areas of Pakistan. World App. Sci. J. 9(12): 1443-1449.

[23] Hussain, M. I., Perveen, A. (2015): Phytosociological attributes of the Plant Biodiversity of the Fort Ranikot and Adjoining Area (Kirthar Range). - Pak. J. Bot. 47(3): 927-935.

[24] Hussain, W., Badshah, L., Ali, A. (2019): Quantitative aspects of the Koh-e-Safaid range vegatation across the altitudinal gradient in upper Kurram valley, Pakistan. - Appl. Ecol. Env. Res. 17(4): 9905-9924.

[25] Ilyas, M., Shinwari, Z. K., Qureshi, R. (2012): Vegetation composition and threats to the montane temperate forest ecosystem of Qalagai hills, Swat, Khyber Pakhtunkhwa, Pakistan. - Pak. J. Bot. 44(SI): 113-122.

[26] Ilyas, M., Qureshi, R., Akhtar, N., Munir, M., Haq, Z. U. (2015): Vegetation analysis of Kabal valley, district Swat, Pakistan using multivariate approach. - Pak. J. Bot. 47(SI): $77-86$.

[27] Irshad, M., Khan, N., Ali, K., Muhammad, Z. (2016): The influence of environmental variables on Punica granatum assemblages in subtropical dry temperate woodland in the district of Dir Lower, Khyber Pakhtunkhwa, Pakistan. - Turk. J. Bot. 40: 610-622.

[28] Khan, N., Ahmad, M., Wahab, M., Nazim, K., Ajaib, M. (2010a): Phytosociology, structure and physicochemical analysis of soil in Quercus baloot Griff. Forest District Chitral, Pakistan. - Pak. J. Bot. 42(4): 2429-2441.

[29] Khan, N., Ahmed, M., Wahab, M., Nazim, K., Ajaib, M. (2010b): Phytosociology, structure and physiochemical analysis of soil in Quercus baloot griff forest, District Chitral, Pakistan. - Pak. J. Bot. 42(4): 2429-2441.

[30] Khan, N., Ahmed, M., Wahab, M., Ajaib, M., Hussain, S. S. (2010c): Studies along an altitudinal gradient in Monotheca buxifolia forest, district Dir Lower, Pakistan. - Pak. J. Bot. 42(5): 3029-3038.

[31] Khan, S. M., Harper, D., Page, S., Ahmad, H. (2011): Species and community diversity of vascular flora along environmental gradients in Naran Valley. A Multivariate approach through indicator species. - Pak. J. Bot. 43(5): 2337-2346.

[32] Khan, S. M. (2012): Plant communities and vegetation ecosystem services in the Naran valley, Western Himalaya. - (Unpublished) Ph.D Thesis, Department of Biological sciences, University of Leicester, 271p. 
[33] Khan, N., Ahmed, M., Siddiqui, M. F., Bibi, S., Ahmed, I. (2012): A phytosociological study of forest and non-forest vegetation of District Chitral, Hindukush Range, Pakistan. - FUUAST J. Bio. 2(1): 91-101.

[34] Khan, A., Ahmad, M., Siddiqui, M. F., Iqbal, J., Wahab, M. (2016): Phytosociological analysis of Pine forest at Indus Kohistan, KPK, Pakistan. - Pak. J. Bot. 48(2): 575-580.

[35] Kjeldahl, J. (1883): A new method for the estimation of nitrogen in organic compounds. Z. Anal. Chem. 22: 366-382.

[36] Kucharik, C. J., Foley, J. A., Delire, C., Fisher, V. A., Coe, M. T., Lenters, J. D., YoungMolling, C., Ramankutty, N. (2000): Testing the performance of a dynamic global ecosystem model: water balance, carbon balance, and vegetation structure. - Glob. Biogeochem. Cycles 14: 795-825.

[37] Liu, H. Y. (2008): Studies of vegetation-environment relationships and vegetation dynamics in Chinese subtropical forests. - Ph.D. Thesis University of Oslo, Department of Botany and Natural History Museum Faculty of Mathematics and Natural Sciences.

[38] Mehmood, A., Khan, S. M., Shah, A. H., Ahmad, H. (2015): First floristic exploration of the district Torghar, Khyber Pakhtunkhwa, Pakistan. - Pak. J. Bot. 47(SI): 57-70.

[39] Menhinick, E. F. (1964): A Comparison of some species diversity indices applied to samples of field insects. - Ecology 45: 859-861.

[40] Mohib Shah, R. (2013): Phytosociological attributes and phytodiversity of Dheri baba hills and Peer Taab Graveyard district Swabi, Khyber Pakhtunkhwa, Pakistan. - J. Life Sci. 1(1): 1-16.

[41] Motyka, J., Dobrzanski, B., Zawadski, S. (1950): Wstepne badania and lakami polundnlowowschodneij Lubeiszczyzny. - Ann. Univ. M. Curie-Jklodowska 13: 367-447. (Sec. E. 5).

[42] Muhammad, Z., Khan, N., Ullah, A. (2015): Temporal trends in phenology and demographic status of Acacia modesta population in Malakand division, Pakistan. - J. Bio. Env. Sci. 6(2): 8-15.

[43] Naveed, A. (2014): Exploring Patterns of Phytodiversity, Ethnobotany, Plant Geography and Vegetation in the Mountains of Miandam, Swat, Northern Pakistan. - Ph.D Thesis, Göttingen.

[44] Olsen, S. R., Sommers, L. E. (1982): Phosphorus. - Methods of Soil Analysis, Part 2 (2 ${ }^{\text {nd }}$ ed.) Medison, WI, USA, pp. 406-407.

[45] Oswalt, S. N., Brandies, T. J., Dimick, B. P. (2006): Phytosociology of vascular plants on an international biosphere reserve: Virgin Islands National Park, St. John, US Virgin Islands. - Caribb. J. Sci. 42(1): 53-66.

[46] Pichi-Sermolli, R. E. (1948): An index for establishing the degree of maturity in plant communities. - J. Ecology 36: 85-90.

[47] Rahman, K., Khan, A., Nisar, M., Hameed, T., Khan, M. S., Jan, A. U., Shariatullah, Ahmad, A., Iqbal, A., Khan, I. A. (2016): Phytosociological study on Isodon rugosus dominated communities in Khwazakhela district Swat, Khyber Pakhtunkhwa, Pakistan. Int. J. Bio 9(6): 292-302.

[48] Rashid, A., Swati, M. F., Sher, H., Al-Yemeni. M. N. (2011): Phytoecological evaluation with detail floristic appraisal of the vegetation around Malam Jabba, Swat, Pakistan. Asian. Pac. J. Trop. Biomed. 1(6): 461-467.

[49] Rayan, J. N., Harvey, R. W., Metge, D. W., Larson, J. E. (1997): Transport of bacteriophage PRDI and silica colloids in a sewage contaminated aquifer. - Eos, Transaction of the American Geophysical Union 86, F 231, Presented at the Fall Meeting of the American Geophysical Union.

[50] Shaheen, H., Ahmad, N., Alam, N., Ahmed, K., Ullah, Z. (2011): Phytodiversity and endemic richness in high altitude Rama Valley, Western Himalayas, Northern Pakistan. J. Med. Pl. Res 5(8): 1489-1493. 
[51] Sharma, P., Rana, J. C., Devi, U. S., Randhawa, S., Kumar, R. (2014): Floristic diversity and distribution pattern of plant communities along altitudinal gradient in Sangla valley, Northwest Himalaya. - Sci. World. J. http://dx.doi.org/10.1155/2014/264878.

[52] Siddiqui, M. F., Ahmed, M. S., Hussain, S. S., Shaukat, S., Khan, N. (2011): Vegetation description and current status of moist temperate coniferous forests of Himalayan and Hindukush region of Pakistan. - Fuuast J. Biol. 1(2): 99-114.

[53] Sorensen, T. (1948): A method of establishing groups of equal amplitudes in plant sociology based on similarity of species content and its application to analyze the vegetation of Darnish commons. - Biol. Skr. 5: 1-34.

[54] Srivastava, R., Mishra, R. M., Awasthi, A. (2016): Phytosociological studies on certain plants of Awarpur (M.S). - Int. J. Pharm. Life. Sci. 7(3): 4930-4936.

[55] Tsiourlis, G., Konstantinids, P., Xofis, P. (2009): Syntaxonomy and Synecology of Quercus coccifera Mediterranean Shrublands in Greece. - J. Pl. Bio. 52(5): 433-447.

[56] Ummara, U., Bokhari, T. Z., Siddiqui, M. F., Dasti, A. A., Younis, U., Hasnain, M., Raza, S., Siddiqui, A. (2015): Quantitative description of understory vegetation of Shogran valley, Pakistan. - Fuuast J. Bio. 5(1): 63-70.

[57] Wahab, M., Ahmed, M., Khan, N., Sarangzai, A. M. (2010): A phytosociological study of pine forests from district Dir, Pakistan. - Int. J. Biol. Biotech. 7(3): 219-226.

[58] Walkley, A. (1947): A critical examination of a rapid method for determining organic carbon in soils: Effect of variation in digestion conditions and of inorganic soil constituents. - Soil. Sci. 63(4): 251-264.

[59] Zhang, J. T., Zhang, F. (2011): Ecological relations between forest communities and environmental variables in the Lishan mountain nature reserve, China. - Afr. J. Agric. Res. 6(2): 248-259. 